\title{
Lead Evolution in Development of an Anti-allergic Agent ; Dimethyl(2-phenoxyethyl)sulfonium p-Toluenesulfonate as the Secondary Lead Compound
}

\author{
Yukio Tada $^{\mathrm{a}^{*}}$, Ichiro Yamawaki ${ }^{\mathrm{a}}$, Shuichi Ueda ${ }^{\mathrm{b}}$, Hiroshi Matsumoto ${ }^{\mathrm{b}}$, \\ Naosuke Matsuura ${ }^{\mathrm{b}}$, Mitsugi Yasumoto ${ }^{\mathrm{a}}$, Akihide Koda ${ }^{\mathrm{c}}$, Mikio Hori ${ }^{\mathrm{c}}$ \\ ${ }^{a}$ Hanno Research Center, Taiho Pharmaceutical Company, Ltd., \\ 1-27 Misugidai, Hanno, Saitama 357-8527, Japan \\ ${ }^{b}$ Biological Laboratory, Taiho Pharmaceutical Company, Ltd., \\ Hiraishi, Ebisuno, Kawauchi-cho, Tokushima 771-0132, Japan \\ ${ }^{c}$ Gifu Pharmaceutical University, \\ 5-6-1 Mitahora-higashi, Gifu 502-0004 Japan \\ *E-mail:tada@taiho.co.jp
}

(Received April 23, 2002; accepted September 4, 2002 ; published online October 22, 2002 )

\begin{abstract}
Derivatives of 2-hydroxyethyldimethylsulfonium $p$-toluenesulfonate (1) were evaluated for anti-allergic activity in order to develop the drug for treatment of type $I$ allergic diseases. The IgE-induced rat homologous passive cutaneous anaphylaxis (PCA) was inhibited by some 2-alkoxy and 2-aryloxyethyl sulfonium $p$-toluenesulfonates. There were some correlations between hydrophobicity or electronic properties (HOMO or charge at the $O$ atom) and anti-allergic activity or acute toxicity. Dimethyl(2-phenoxyethyl)sulfonium $p$-toluenesulfonate (5) was selected as a second lead compound in the next stage.
\end{abstract}

Key Words: lead evolution, antialllergic activity, sulfonium $p$-toluenesulfonate, QSAR

Area of Interest: Molecular Recognition

\section{Introduction}

Type I allergic disorders such as bronchial asthma, atopic dermatitis, and allergic rhinitis are mediated by allergen-specific IgE antibodies. Therefore, a drug that specifically inhibits $\operatorname{IgE}$ antibody formation will be expected to be efficacious for the fundamental treatment of type I allergic diseases. Our purpose is to obtain such compounds that exhibit a specific suppression of the type I allergic reaction without decreasing the production of $\operatorname{IgM}$ and $\operatorname{IgG}$ hemolytic plaque forming cells (HPFC) in mice immunized with sheep red blood cells (SRBC) [1].

We examined the immunological activity of several kinds of sulfonium compounds and chose 
2-hydroxyethyldimethylsulfonium p-toluenesulfonate (1) as a lead compound that increased the production of HPFC [2]. Following that, we examined some derivatives of compound 1 by further immunological studies in to find improved the lead compounds that have anti-allergic activity in type I allergic reaction [3].

\section{Lead Evolution from the First Lead Compound ; 2-Hydroxyethyl- dimethylsulfonium $p$-Toluenesulfonate (1)}

IgE-induced 48-hr homologous passive cutaneous anaphylaxis (PCA) is a model for type I allergic reaction [4]. Compound 1 increased the production of HPFC [2]; however, it showed little PCA inhibitory activity. In contrast, a methoxy derivative (2) having some PCA inhibitory activity was found among the various derivatives of compound 1. Some alkoxy $(3,4)$, and phenoxy (5-7) derivatives were tested for their PCA inhibitory activity (Table 1). In this testing 2-phenoxyethyl derivative (5) showed $72.7 \%$ inhibition. This inhibitory activity is very strong compared with that of Tranilast, an anti-allergic drug [5]. Accordingly, we selected dimethyl(2-phenoxyethyl)sulfonium $p$-toluenesulfonate (5) as a candidate for the secondary lead compound derived from the first lead compound (1).

Table 1. LD50(ip)a and PCA Inhibition (\%)b

\begin{tabular}{|c|c|c|c|c|c|c|}
\hline No & Sulfonium Cation & $\Sigma \pi_{\text {i calcd }}$ & $\mathrm{HOMO}(\mathrm{eV})$ & charge & $\mathrm{LD}_{50}(\mathrm{mmol} / \mathrm{kg})^{\mathrm{a}}$ & PCA inhibition (\%) \\
\hline 1 & $\left(\mathrm{CH}_{3}\right)_{2} \mathrm{~S}^{+} \mathrm{CH}_{2} \mathrm{CH}_{2} \mathrm{OH}$ & 0.43 & - & - & 4.69 & 5.7 \\
\hline 2 & $\left(\mathrm{CH}_{3}\right)_{2} \mathrm{~S}^{+} \mathrm{CH}_{2} \mathrm{CH}_{2} \mathrm{OCH}_{3}$ & 0.74 & -14.49 & -0.263 & 1.11 & 15.1 \\
\hline 3 & $\left(\mathrm{CH}_{3}\right)_{2} \mathrm{~S}^{+} \mathrm{CH}_{2} \mathrm{CH}_{2} \mathrm{O}$ & 2.21 & -13.46 & -0.243 & 1.27 & 19.7 \\
\hline 4 & $\left(\mathrm{CH}_{3}\right)_{2} \mathrm{~S}^{+} \mathrm{CH}_{2} \mathrm{CH}_{2} \mathrm{O}-$ & 2.77 & -13.19 & -0.259 & 0.79 & 41.8 \\
\hline 5 & $\left(\mathrm{CH}_{3}\right)_{2} \mathrm{~S}^{+} \mathrm{CH}_{2} \mathrm{CH}_{2} \mathrm{O}$ & 2.99 & -12.48 & -0.217 & 0.52 & 72.7 \\
\hline 6 & $\left(\mathrm{CH}_{3}\right)_{2} \mathrm{~S}^{+} \mathrm{CH}_{2} \mathrm{CH}_{2} \mathrm{O}$ & 4.01 & -11.81 & -0.215 & 0.43 & 30.5 \\
\hline \multirow[t]{2}{*}{7} & $\left(\mathrm{CH}_{3}\right)_{2} \mathrm{~S}^{+} \mathrm{CH}_{2} \mathrm{CH}_{2} \mathrm{O}$ & 4.56 & -11.91 & -0.213 & 0.31 & 23.3 \\
\hline & Tranilast & & & & & 45.1 \\
\hline
\end{tabular}

${ }^{\mathrm{a}} \mathrm{LD}_{50}$ (ip) in mice, ${ }^{\mathrm{b}}$ Dose(ip) $20 \mathrm{mg} / \mathrm{kg}$ in rat, ${ }^{\mathrm{c}}$ Dose(iv) $20 \mathrm{mg} / \mathrm{kg}$ in rat 
To evaluate compound 5, several sulfonium compounds that have the phenoxyethyl moiety were examined by the PCA test (Scheme1, Table 2). Since methylethyl and diethylsulfonium compounds $(\mathbf{8}, 9)$ reduced PCA inhibitory activity, the dimethylsulfinio group is considered to be desirable. To evaluate the length of the methylene of compound $\mathbf{5}$, we examined phenoxypropyl (10), butyl (11), and pentyl (12) derivatives, as well as, 1- or 2- methylethyl (13, 14) and pentahydrothiopyranyl (15) derivatives, but found that they were no more active than compound $\mathbf{5}$. Thus, a dimethylsulfonio group is desirable and an ethylene moiety is a desirable linker between the sulfonio and phenoxy groups.

\section{Scheme 1}

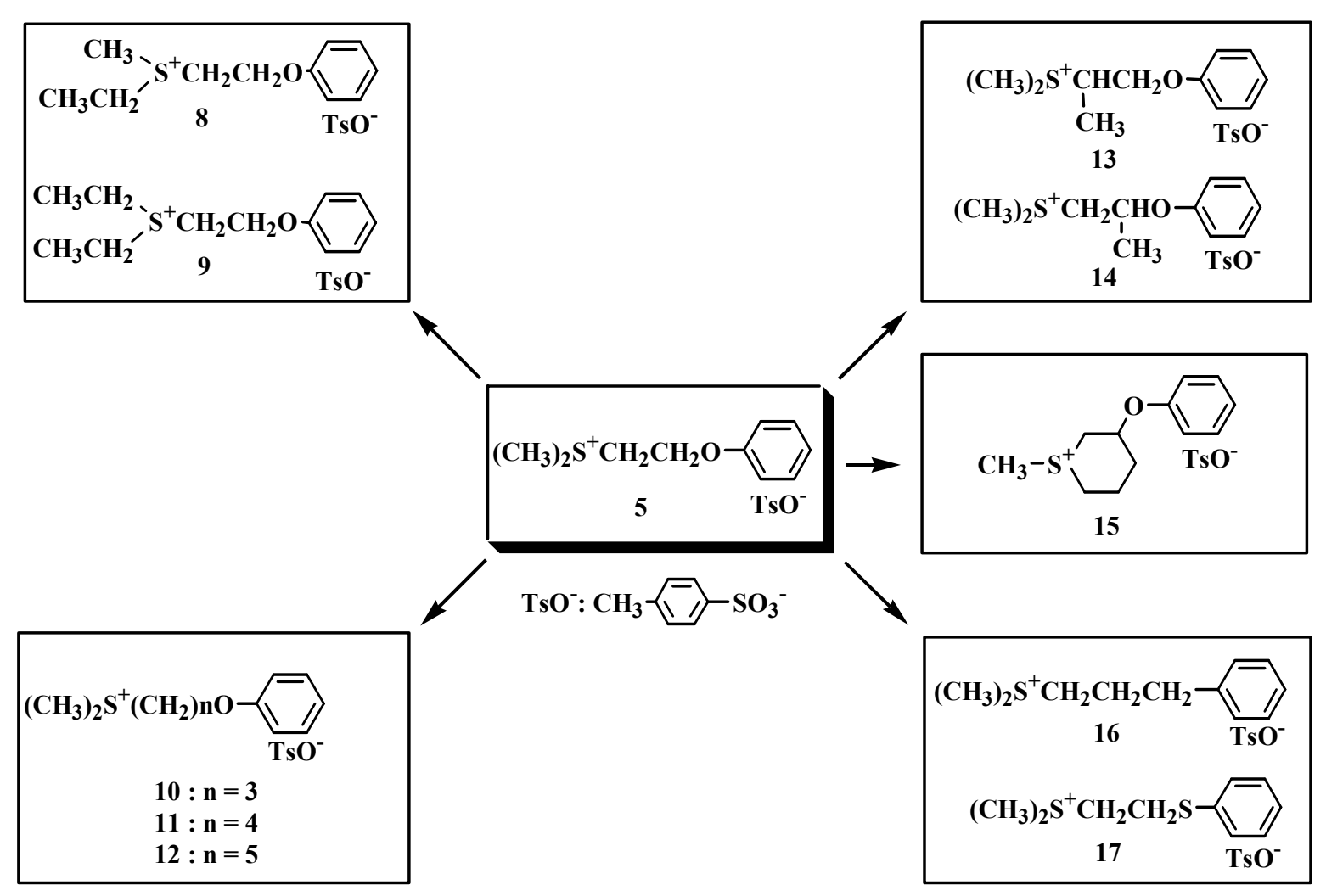

On the other hand, a 3-Phenypropyl derivative (16) that has no ether $\mathrm{O}$ atom had little activity, and a 2-phenylthioethyl derivative (17) that has an $\mathrm{S}$ atom instead of an $\mathrm{O}$ atom had no activity, and thus the ether $\mathrm{O}$ atom of the phenoxyethyl group is important for PCA inhibitory activity. These results suggest that the 2-phenoxyethyldimethylsulfonio moiety would be desirable for PCA inhibitory activity.

To confirm the PCA inhibitory activity of sulfonium $p$-toluenesulfonate itself, methylsulfide (18), methylsulfoxide (19), and methysulfone (20) derivatives were tested [6]. These compounds (18-20) had weak PCA inhibitory activity (Scheme 2). Consequently, we finally decided on dimethyl(2-phenoxyethyl)sulfonium $p$-toluenesulfonate (5) as a secondary lead compound for lead optimization in the next stage. 
Table 2. LD50(ip)a and PCA Inhibition (\%)b

\begin{tabular}{|c|c|c|c|c|c|c|}
\hline No & Sulfonium Cation & $\Sigma \pi_{\mathrm{i} \mathrm{calcd}}$ & \multicolumn{3}{|c|}{$\mathrm{HOMO}(\mathrm{eV})$ charge LD50 $(\mathrm{mmol} / \mathrm{kg})^{\mathrm{a}}$} & PCA inhibition $(\%)^{b}$ \\
\hline 8 & $\mathrm{CH}_{3} \mathrm{H}_{3} \mathrm{CH}_{2}^{\prime} \mathrm{S}$ & 3.44 & -12.12 & -0.213 & - & 29.5 \\
\hline 9 & $\begin{array}{l}\mathrm{H}_{3} \mathrm{CH}_{2} \\
\mathrm{H}_{3} \mathrm{CH}_{2}^{\prime} \mathrm{S}^{+} \mathrm{C}\end{array}$ & 3.89 & -11.88 & -0.203 & - & 18.7 \\
\hline 10 & $\left(\mathrm{CH}_{3}\right)_{2} \mathrm{~S}^{+}(\mathrm{C}$ & 3.52 & -11.81 & -0.232 & 0.53 & 30 \\
\hline 11 & $\left(\mathrm{CH}_{3}\right)_{2} \mathrm{~S}^{+}\left(\mathrm{CH}_{2}\right)_{4}$ & 4.05 & -11.11 & -0.196 & 0.25 & -3.9 \\
\hline 12 & $\left(\mathrm{CH}_{3}\right)_{2} \mathrm{~S}^{+}\left(\mathrm{CH}_{2}\right)_{5}$ & 4.58 & -10.94 & -0.195 & 0.19 & 14.8 \\
\hline 13 & $\begin{array}{c}\left(\mathrm{CH}_{3}\right)_{2} \mathrm{~S}^{+} \underset{\mathrm{I}}{\mathrm{C} H C \mathrm{H}_{2} \mathrm{O}} \\
\stackrel{\mathrm{C} \mathrm{H}_{3}}{ }\end{array}$ & 3.39 & -12.11 & -0.214 & 0.31 & 31.5 \\
\hline 14 & $\left(\mathrm{CH}_{3}\right)_{2} \mathrm{~S}^{+} \mathrm{CH}$ & 3.39 & -12.14 & -0.168 & 0.64 & 36.2 \\
\hline 15 & & 3.39 & -11.99 & -0.166 & 0.13 & 42 \\
\hline 16 & $\left.\mathrm{H}_{3}\right)_{2} \mathrm{~S}^{+} \mathrm{CH}_{2} \mathrm{C}$ & 3.43 & -11.98 & -0.038 & 0.24 & 26.1 \\
\hline 17 & $\left(\mathrm{CH}_{3}\right)_{2} \mathrm{~S}^{+} \mathrm{CH}_{2} \mathrm{CH}_{2} \mathrm{~S}$ & 4.05 & -12.41 & 0.076 & 0.25 & -29.2 \\
\hline
\end{tabular}

${ }^{\mathrm{a}} \mathrm{LD}_{50}$ (ip) in mice, ${ }^{\mathrm{b}}$ Dose(ip) $20 \mathrm{mg} / \mathrm{kg}$ in rat

\section{Scheme 2}
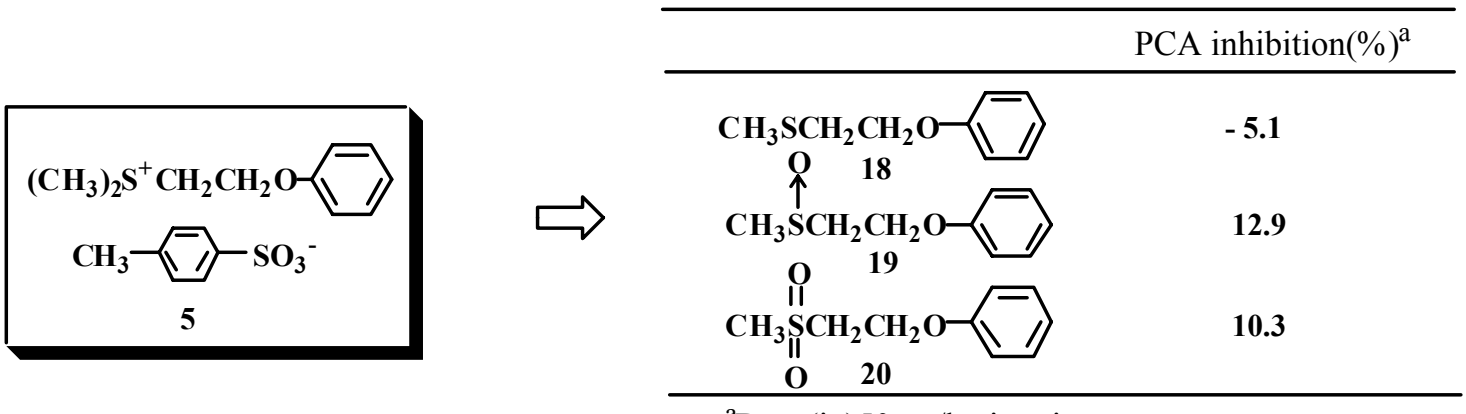

${ }^{\mathrm{a}}$ Dose(ip) $50 \mathrm{mg} / \mathrm{kg}$ in mice 


\section{Structure Activity Relationships in PCA Inhibitory Activity and Acute Toxicity}

Six of the sulfonium compounds (4-6, 13-15) have strong PCA inhibitory activity, although we have little information about their target proteins and inhibitory mechanisms. Therefore, physicochemical properties are the only information available for molecular modeling. We examined the hydrophobicity and some electronic properties that can be calculated by MOPAC to obtain some correlation between the physicochemical properties and PCA inhibitory activity and acute toxicity. The hydrophobic parameters of the substituent $\left(\pi_{\text {calcd }}\right)$ of $\mathrm{S}^{+}$were calculated by C $\log \mathrm{P}\left(\right.$ ver. 2.0.0) [7], and $\Sigma \pi_{\mathrm{i} \text { calcd }}$ represents the summation of $\pi_{\text {calcd. }}$ Logit (PCA): $\log$ [PCA inhibition \% / (100 - PCA inhibition \%)] was used as the dependent variable. There is a correlation between the PCA inhibitory activity and hydrophobicity $\left(\Sigma \pi_{\mathrm{i} \text { calcd }}\right)$ for the 15 compounds (1-10, 12-16) that had some inhibitory activity. (eq 1, Figure1). In this and the following equations, $\mathrm{n}$ is the number of compounds in each series, $\mathrm{r}$ is the correlation coefficient, $\mathrm{s}$ is the standard deviation, and $\mathrm{F}$ is the ratio between regression and residual variances. The figures in parentheses are the $95 \%$ confidence intervals of the regression coefficient and the intercept. The correlation coefficient of eq (2) is not good, but this equation is statistically significant. From eq (1), the optimum $\Sigma \pi_{\mathrm{i} \text { calcd }}$ is suggested to be about 2.88 .

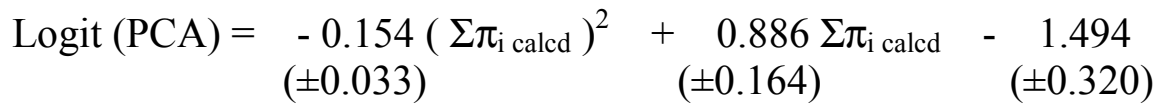

$$
\begin{aligned}
& (\mathrm{n}=15, \mathrm{r}=0.778, \mathrm{~s}=0.246, \mathrm{~F}=9.17)
\end{aligned}
$$

Conversely, increasing the hydrophobicity of substituents tends to strengthen the acute toxicity (eq 2) of the sulfonium compounds (1-7, 10-17).

$$
\begin{aligned}
& \log \left(1 / \mathrm{LD}_{50}\right)=\underset{( \pm 0.118)}{0.254 \Sigma \pi_{\mathrm{i} \text { calcd }}}-\begin{array}{c}
0.456 \\
( \pm 0.396)
\end{array} \\
& (\mathrm{n}=15, \mathrm{r}=0.789, \mathrm{~s}=0.250, \mathrm{~F}=21.42)
\end{aligned}
$$

In addition to hydrophobicity, the electronic properties of sulfonium compounds were considered, to llok for some correlation with their biological activities. 2-Phenoxyethytrimethyl ammonium bromide is a isostere of compound $\mathbf{5}$, and its electronic structure and nicotine-like stimulatory activity have been reported [9]. The molecular orbital calculations of such electronic parameters as electronic energy, atomic charge at $\mathrm{O}$, heat of formation, HOMO, LUMO eigenvalues, super delocalizability at the ortho position of the phenoxy group, and total dipole, of these compounds (1-7, 10-17) were performed by using the MOPAC/PM3 [10]. There exists a strong

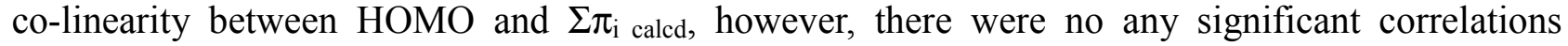
between the electronic properties and PCA inhibitory activity. But HOMO and the atomic charge at the $\mathrm{O}$ atom were found to correlate with acute toxicity (eq 3,4). The eqs (3) and (4) suggest that a lower HOMO eigenvalue and larger negative charge at $\mathrm{O}$ are favorable from the standpoint of acute toxicity. 


$$
\begin{aligned}
& \log \left(1 / \mathrm{LD}_{50}\right)=\underset{( \pm 0.089)}{0.276 \mathrm{HOMO}}+\begin{array}{c}
3.778 \\
( \pm 1.333)
\end{array} \\
& (\mathrm{n}=15, \mathrm{r}=0.881, \mathrm{~s}=0.193, \mathrm{~F}=44.95) \\
& \log \left(1 / \mathrm{LD}_{50}\right)=8.680 \text { charge }+2.210 \\
& ( \pm 4.500) \quad( \pm 1.526) \\
& (\mathrm{n}=13, \mathrm{r}=0.854, \mathrm{~s}=0.220, \mathrm{~F}=29.80)
\end{aligned}
$$

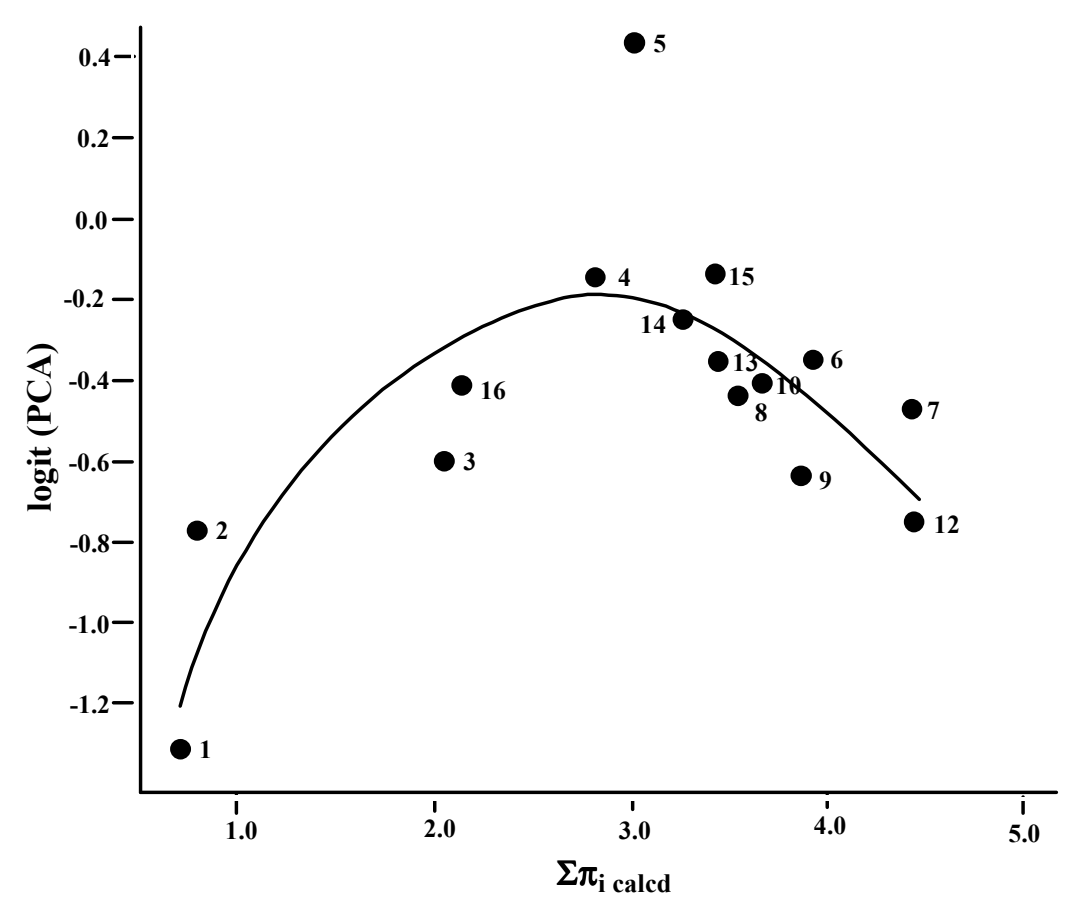

Figure 1. Plot of $\Sigma \pi_{\mathrm{i} \text { calcd }}$ vs. $\operatorname{logit}(\mathrm{PCA})$.

\section{Conclusion}

Dimethyl(2-phenoxyethyl)sulfonium $p$-toluenesulfonate (5) that has a negatively charged $\mathrm{O}$ atom in the phenoxyethyl moiety in addition to the sulfonium head $\left(\mathrm{S}^{+}\right)$was selected as the secondary lead compound, which was derived from the first lead compound 2-hydroxydimethylsulfonium $p$-toluenesulfonate (1). In implementing the lead optimization, it is suggested that about 3.00 hydrophobic parameters of the substituent $\Sigma \pi_{\text {i calcd }} 2.88$ are desirable for PCA inhibitory activity, and the HOMO eigenvalue and charge of the $\mathrm{O}$ atom of the phenoxyethyl moiety should be considered from the point of toxicity. 


\section{Experimental}

\subsection{Synthesis of Sulfonium p-toluenesulfonates}

Melting points were determined with a Yanagimoto MP-3 micro melting point apparatus and are uncorrected. ${ }^{1} \mathrm{H}$ NMR spectra were obtained in DMSO- $\mathrm{d}_{6}$ with $\mathrm{Me}_{4} \mathrm{Si}$ as internal standard on a JEOL LMN-FX 100 spectrometer. Analytical results for compounds followed by elemental symbols were determined on a Perkin-Elmer Model $240 \mathrm{CHN}$ analyzer. 2-Hydroxyethyldiemethylsulfonium (1), 2-methoxyethyldimethylsulfonium (2), sulfide (18), sulfoxide (19) and sulfone derivatives (20) were prepared as described in the references[2,5]. Sulfonium p-toluenesulfonate derivatives (3, 6-17) were prepared in the same manner as for compound 4 or 5.

2-Cyclohexyloxyethyldimethylsulfonium $\boldsymbol{p}$-toluenesulfonate (4): Methanethiol (15.0 g, 0.31 mol) was dissolved in a solution of sodium $(6.0 \mathrm{~g}, 0.26 \mathrm{~mol})$ in $\mathrm{EtOH}(100 \mathrm{~mL})$ and 2-cyclohexyl ethyl chloride $(35 \mathrm{~g}, 0.22 \mathrm{~mol})$ was added dropwise to the solution. The reaction mixture was stirred for $12 \mathrm{hr}$ at room temperature, and then $\mathrm{EtOH}$ was removed. The residue was extracted with ether $(300 \mathrm{~mL})$. The extracts were washed with water, dried $\left(\mathrm{MgSO}_{4}\right)$, and concentrated to give 2-cyclohexyl ethyl sulfide $(21.3 \mathrm{~g}, 56.9 \%)$. A mixture of obtained sulfide $(5.23 \mathrm{~g}, 0.03 \mathrm{~mol})$ and methyl $p$-toluenesulfonate $(11.17 \mathrm{~g}, 0.06 \mathrm{~mol})$ was stirred for 3 days at room temperature and then ether $(100 \mathrm{~mL})$ was added. A crystallized solid was collected by filtration and was recrystallized from EtOH-ether to give $8.20 \mathrm{~g}(75.8 \%)$ of 4: mp 90-91 ${ }^{\circ} \mathrm{C} ;{ }^{1} \mathrm{H}-\mathrm{NMR}\left(\mathrm{DMSO}-\mathrm{d}_{6}\right)$ \%: $7.49(2 \mathrm{H}, \mathrm{d}$, $\mathrm{J}=7.8 \mathrm{~Hz}$, tosyl $\left.\mathrm{H}_{3,5}\right), 7.11\left(2 \mathrm{H}, \mathrm{d}, \mathrm{J}=7.8 \mathrm{~Hz}\right.$, tosyl $\left.\mathrm{H}_{2,6}\right), 3.82\left(2 \mathrm{H}, \mathrm{m}, \mathrm{S}^{+} \mathrm{CH}_{2} \mathrm{CH}_{2}\right), 3.57(2 \mathrm{H}, \mathrm{m}$, $\left.\mathrm{S}^{+} \mathrm{CH}_{2}\right), 3.33(1 \mathrm{H}$, broad, $\mathrm{OCH}), 2.90\left(6 \mathrm{H}, \mathrm{s},\left(\mathrm{CH}_{3}\right)_{2} \mathrm{~S}^{+}\right), 2.29\left(3 \mathrm{H}, \mathrm{s}\right.$, tosyl $\left.\mathrm{CH}_{3}\right), 0.9 \sim 2.0(10 \mathrm{H}, \mathrm{m}$, cyclohexyl $\mathrm{H}_{2}$ ). Anal Calcd for $\mathrm{C}_{17} \mathrm{H}_{28} \mathrm{O}_{4} \mathrm{~S}_{2}$ : C, 56.64; H, 7.83. Found: C, 56.64; H, 8.02.

2-Cyclopentyloxydimethylsulfonium p-toluenesulfonate (3): $\mathrm{mp} \quad 81-82{ }^{\circ} \mathrm{C}$; ${ }^{1} \mathrm{H}-\mathrm{NMR}$ $\left(\mathrm{DMSO}_{-} \mathrm{d}_{6}\right) \delta: 7.48\left(2 \mathrm{H}, \mathrm{d}, \mathrm{J}=7.8 \mathrm{~Hz}\right.$, tosyl $\left.H_{3,5}\right), 7.11\left(2 \mathrm{H}, \mathrm{d}, \mathrm{J}=7.8 \mathrm{~Hz}\right.$, tosyl $\left.H_{2,6}\right), 3.91(1 \mathrm{H}$, broad, $\mathrm{OCH}), 3.70\left(2 \mathrm{H}, \mathrm{m}, \mathrm{S}^{+} \mathrm{CH}_{2} \mathrm{CH}_{2}\right), 3.50\left(2 \mathrm{H}, \mathrm{m}, \mathrm{S}^{+} \mathrm{CH}_{2}\right), 2.89\left(6 \mathrm{H}, \mathrm{s},\left(\mathrm{CH}_{3}\right)_{2} \mathrm{~S}^{+}\right), 2.20\left(3 \mathrm{H}, \mathrm{s}\right.$, tosyl $\left.\mathrm{CH}_{3}\right)$, 1.2 2.0( $\left(8 \mathrm{H}, \mathrm{m}\right.$, cyclopentyl $\left.\mathrm{CH}_{2}\right)$. Anal Calcd for $\mathrm{C}_{16} \mathrm{H}_{26} \mathrm{O}_{4} \mathrm{~S}_{2}: \mathrm{C}, 55.46 ; \mathrm{H}, 7.56$. Found: $\mathrm{C}, 55.52$; $\mathrm{H}, 7.73$.

Dimethyl(2-phenoxyethyl)sulfonium p-toluenesulfonate (5): A mixture of 2-chloroethyl methyl sulfide $(22.10 \mathrm{~g}, 0.20 \mathrm{~mol})$, phenol $(18.82 \mathrm{~g}, 0.20 \mathrm{~mol})$ and $\mathrm{NaOH}(8.00 \mathrm{~g}, 0.20 \mathrm{~mol})$ in $95 \%$ $\mathrm{EtOH}$ was refluxed for $8 \mathrm{hr}$ and then $\mathrm{EtOH}$ was removed. The residue was extracted with ether (300 $\mathrm{mL})$. The extracts were washed with water, dried $(\mathrm{MgSO} 4)$, and concentrated to give 2-phenoxyethyl methyl sulfide (21.32 g, 63.5\%). A mixture of obtained sulfide (5.05 g, $0.03 \mathrm{~mol}$ ) and methyl $p$-toluenesulfonate $(11.17 \mathrm{~g}, 0.06 \mathrm{~mol})$ was stirred for 3 days at room temperature and then ether $(100 \mathrm{~mL})$ was added. A crystallized solid was collected by filtration and was recrystallized from EtOH-ether to give $6.20 \mathrm{~g}(58.3 \%)$ of 5: $\mathrm{mp} 146-167{ }^{\circ} \mathrm{C}$; ${ }^{1} \mathrm{H}-\mathrm{NMR}$ $\left(\mathrm{DMSO}_{6} \mathrm{~d}_{6}\right) \delta: 7.43\left(2 \mathrm{H}, \mathrm{d}, \mathrm{J}=7.8 \mathrm{~Hz}\right.$, tosyl $\left.H_{3,5}\right), 7.26(2 \mathrm{H}, \mathrm{m}$, aromatic $\mathrm{H}), 7.03(2 \mathrm{H}, \mathrm{d}, \mathrm{J}=7.8 \mathrm{~Hz}$, tosyl $\left.\mathrm{H}_{2,6}\right), 6.92(3 \mathrm{H}, \mathrm{m}$, aromatic $\mathrm{H}), 4.42\left(2 \mathrm{H}, \mathrm{t}, \mathrm{J}=5.3 \mathrm{~Hz}, \mathrm{~S}^{+} \mathrm{CH}_{2} \mathrm{CH}_{2}\right), 3.57(2 \mathrm{H}, \mathrm{t}, \mathrm{J}=5.3 \mathrm{~Hz}$, $\left.\mathrm{S}^{+} \mathrm{CH}_{2}\right), 2.96\left(6 \mathrm{H}, \mathrm{s},\left(\mathrm{CH}_{3}\right)_{2} \mathrm{~S}^{+}\right), 2.25\left(3 \mathrm{H}, \mathrm{s}\right.$, tosyl $\left.\mathrm{CH}_{3}\right)$. Anal Calcd for $\mathrm{C}_{17} \mathrm{H}_{22} \mathrm{O}_{4} \mathrm{~S}_{2}: \mathrm{C}, 57.60 ; \mathrm{H}$, 6.26. Found: C, 57.29; H, 6.39 .

2-(5-Indanyloxy)ethyldimethylsulfonium p-toluenesulfonate (6): $\mathrm{mp}$ 131-132 ${ }^{\circ} \mathrm{C} ;{ }^{1} \mathrm{H}-\mathrm{NMR}$ $\left(\right.$ DMSO-d $\left._{6}\right) \delta: 7.42\left(2 \mathrm{H}, \mathrm{d}, \mathrm{J}=7.8 \mathrm{~Hz}\right.$, tosyl $\left.H_{3,5}\right), 7.05\left(2 \mathrm{H}, \mathrm{d}, \mathrm{J}=7.8 \mathrm{~Hz}\right.$, tosyl $\left.H_{2,6}\right), 6.57 \sim 7.15(3 \mathrm{H}, \mathrm{m}$, aromatic $\mathrm{H}), 4.34\left(2 \mathrm{H}, \mathrm{t}, \mathrm{J}=5.5 \mathrm{~Hz}, \mathrm{~S}^{+} \mathrm{CH}_{2} \mathrm{CH}_{2}\right), 3.70\left(2 \mathrm{H}, \mathrm{t}, \mathrm{J}=5.5 \mathrm{~Hz}, \mathrm{~S}^{+} \mathrm{CH}_{2}\right), 2.90\left(6 \mathrm{H}, \mathrm{s},\left(\mathrm{CH}_{3}\right)_{2} \mathrm{~S}^{+}\right)$, 2.65 2.90(4H, m, $\left.\mathrm{CH}_{2} \mathrm{CH}_{2} \mathrm{CH}_{2}\right), 2.22\left(3 \mathrm{H}, \mathrm{s}\right.$, tosyl $\left.\mathrm{CH}_{3}\right), 1.98\left(2 \mathrm{H}, \mathrm{m}, \mathrm{CH}_{2} \mathrm{CH}_{2} \mathrm{CH}_{2}\right)$. Anal Calcd for $\mathrm{C}_{20} \mathrm{H}_{26} \mathrm{O}_{4} \mathrm{~S}_{2}$ : C, 60.89; H, 6.64. Found: C, 60.81; H, 6.73.

2-[2-(5,6,7,8-Tetrahydronaphthyloxy)]ethylsulfonium $\quad \boldsymbol{p}$-Toluenesulfonate $\quad(7): \quad \mathrm{mp}$ 146-167 ${ }^{\circ}$; ${ }^{1} \mathrm{H}-\mathrm{NMR}\left(\mathrm{DMSO}_{\mathrm{d}}\right) \delta$ : $7.49\left(2 \mathrm{H}, \mathrm{d}, \mathrm{J}=7.8 \mathrm{~Hz}\right.$, tosyl $\left.H_{3,5}\right), 7.10(2 \mathrm{H}, \mathrm{d}, \mathrm{J}=7.8 \mathrm{~Hz}$, tosyl 
$\left.H_{2,6}\right), 6.65 \sim 7.02(3 \mathrm{H}, \mathrm{m}$, aromatic $H), 4.26\left(2 \mathrm{H}, \mathrm{t}, \mathrm{J}=5.5 \mathrm{~Hz}, \mathrm{~S}^{+} \mathrm{CH}_{2} \mathrm{CH}_{2}\right), 3.71(2 \mathrm{H}, \mathrm{t}, \mathrm{J}=5.5 \mathrm{~Hz}$, $\left.\mathrm{S}^{+} \mathrm{CH}_{2}\right), 2.96\left(6 \mathrm{H}, \mathrm{s},\left(\mathrm{CH}_{3}\right)_{2} \mathrm{~S}^{+}\right), 2.65\left(4 \mathrm{H}, \mathrm{m}, \mathrm{CH}_{2} \mathrm{CH}_{2} \mathrm{CH}_{2} \mathrm{CH}_{2}\right), 2.26\left(3 \mathrm{H}\right.$, s, tosyl $\left.\mathrm{CH}_{3}\right), 1.70(4 \mathrm{H}, \mathrm{m}$, $\mathrm{CH}_{2} \mathrm{CH}_{2} \mathrm{CH}_{2} \mathrm{CH}_{2}$ ). Anal Calcd for $\mathrm{C}_{21} \mathrm{H}_{28} \mathrm{O}_{4} \mathrm{~S}_{2}$ : C, 61.73; H, 6.91. Found: $\mathrm{C}, 61.78 ; \mathrm{H}, 6.82$.

Ethylmethyl(2-phenoxyethyl)sulfonium p-toluenesulfonate (8): $\mathrm{mp} \quad 76-77{ }^{\circ} \mathrm{C} ;{ }^{1} \mathrm{H}-\mathrm{NMR}$ $\left(\mathrm{DMSO}_{-}\right)_{6} \delta: 7.51\left(2 \mathrm{H}, \mathrm{d}, \mathrm{J}=7.8 \mathrm{~Hz}\right.$, tosyl $\left.H_{3,5}\right), 6.91 \sim 7.45(5 \mathrm{H}, \mathrm{m}$, aromatic $H), 7.11(2 \mathrm{H}, \mathrm{d}$, $\mathrm{J}=7.8 \mathrm{~Hz}$, tosyl $\left.\mathrm{H}_{2,6}\right), 4.45\left(2 \mathrm{H}, \mathrm{t}, \mathrm{J}=5.3 \mathrm{~Hz}, \mathrm{~S}^{+} \mathrm{CH}_{2} \mathrm{CH}_{2}\right), 3.79\left(2 \mathrm{H}, \mathrm{t}, \mathrm{J}=5.3 \mathrm{~Hz}, \mathrm{~S}^{+} \mathrm{CH}_{2}\right), 3.44(2 \mathrm{H}, \mathrm{q}$, $\left.\mathrm{J}=7.4 \mathrm{~Hz}, \mathrm{CH}_{2} \mathrm{~S}^{+}\right), 2.98\left(3 \mathrm{H}, \mathrm{s}, \mathrm{CH}_{3} \mathrm{~S}^{+}\right), 2.28\left(3 \mathrm{H}, \mathrm{s}\right.$, tosyl $\left.\mathrm{CH}_{3}\right), 1.37\left(3 \mathrm{H}, \mathrm{t}, \mathrm{J}=7.4 \mathrm{~Hz}, \mathrm{CH}_{3} \mathrm{CH}_{2} \mathrm{~S}^{+}\right)$. Anal Calcd for $\mathrm{C}_{18} \mathrm{H}_{24} \mathrm{O}_{4} \mathrm{~S}_{2}$ : C, 58.67; H, 6.56. Found: C, 58.50; H, 6.43.

Diethyl(2-phenoxyethyl)sulfonium p-toluenesulfonate $\quad(9): \quad \mathrm{mp} \quad 56-57 \quad{ }^{\circ} \mathrm{C} ; \quad{ }^{1} \mathrm{H}-\mathrm{NMR}$ $\left(\right.$ DMSO-d $\left._{6}\right) \delta: 7.52\left(2 \mathrm{H}, \mathrm{d}, \mathrm{J}=7.8 \mathrm{~Hz}\right.$, tosyl $\left.H_{3,5}\right), 6.73 \sim 7.43(5 \mathrm{H}, \mathrm{m}$, aromatic $H), 7.12(2 \mathrm{H}, \mathrm{d}$, $\mathrm{J}=7.8 \mathrm{~Hz}$, tosyl $\left.\mathrm{H}_{2,6}\right), 4.31\left(2 \mathrm{H}, \mathrm{t}, \mathrm{J}=5.3 \mathrm{~Hz}, \mathrm{~S}^{+} \mathrm{CH}_{2} \mathrm{CH}_{2}\right), 3.81\left(2 \mathrm{H}, \mathrm{t}, \mathrm{J}=5.3 \mathrm{~Hz}, \mathrm{~S}^{+} \mathrm{CH}_{2}\right), 3.46(4 \mathrm{H}, \mathrm{q}$, $\left.\mathrm{J}=7.3 \mathrm{~Hz}, 2 \times \mathrm{CH}_{2} \mathrm{~S}^{+}\right), 2.28\left(3 \mathrm{H}\right.$, s, tosyl $\left.\mathrm{CH}_{3}\right), 1.37\left(6 \mathrm{H}, \mathrm{t}, \mathrm{J}=7.3 \mathrm{~Hz}, 2 \times \mathrm{CH}_{3} \mathrm{CH}_{2} \mathrm{~S}^{+}\right)$. Anal Calcd for $\mathrm{C}_{19} \mathrm{H}_{26} \mathrm{O}_{4} \mathrm{~S}_{2}$ : C, 59.66; H, 6.85. Found: C, 59.53; H, 6.79.

Dimethyl(3-phenoxypropyl)sulfonium $p$-toluenesulfonate (10): $\mathrm{mp} \quad 160-161{ }^{\circ} \mathrm{C} ;{ }^{1} \mathrm{H}-\mathrm{NMR}$ $\left(\mathrm{DMSO}_{-}\right)$$)$: 7.51(2H, d, J=7.8Hz, tosyl $\left.H_{3,5}\right), 7.17\left(2 \mathrm{H}, \mathrm{d}, \mathrm{J}=7.8 \mathrm{~Hz}\right.$, tosyl $\left.H_{2,6}\right), 7.24 \sim 7.40(5 \mathrm{H}, \mathrm{m}$, aromatic H), 3.30(2H, t, J=7.3Hz, $\left.\mathrm{CH}_{2} \mathrm{O}\right), 2.69\left(2 \mathrm{H}, \mathrm{t}, \mathrm{J}=7.3 \mathrm{~Hz}, \mathrm{~S}^{+} \mathrm{CH}_{2}\right), 2.88\left(6 \mathrm{H}, \mathrm{s},\left(\mathrm{CH}_{3}\right)_{2} \mathrm{~S}^{+}\right)$, 2.28(3H, s, tosyl $\mathrm{CH} 3), 1.80 \sim 2.20\left(2 \mathrm{H}, \mathrm{m}, \mathrm{CH}_{2} \mathrm{CH}_{2} \mathrm{CH}_{2}\right)$. Anal Calcd for $\mathrm{C}_{18} \mathrm{H}_{24} \mathrm{O}_{4} \mathrm{~S}_{2}: \mathrm{C}, 58.67$; H,6.56. Found: C, 58.67; H, 6.62.

Dimethyl(4-phenoxybutyl)sulfonium p-toluenesulfonate (11): $\mathrm{mp} \quad 122-123 \quad{ }^{\circ} \mathrm{C}$; ${ }^{1} \mathrm{H}-\mathrm{NMR}$ $\left(\right.$ DMSO-d $\left._{6}\right) \delta: 7.35\left(2 \mathrm{H}, \mathrm{d}, \mathrm{J}=7.8 \mathrm{~Hz}\right.$, tosyl $\left.H_{3,5}\right), 7.05\left(2 \mathrm{H}, \mathrm{d}, \mathrm{J}=7.8 \mathrm{~Hz}\right.$, tosyl $\left.H_{2,6}\right), 6.70 \sim 7.25(5 \mathrm{H}, \mathrm{m}$, aromatic H), 3.84(2H, t, J=6.0Hz, $\left.\mathrm{CH}_{2} \mathrm{O}\right), 3.20\left(2 \mathrm{H}, \mathrm{t}, \mathrm{J}=6.0 \mathrm{~Hz}, \mathrm{~S}^{+} \mathrm{CH}_{2}\right), 2.75\left(6 \mathrm{H}, \mathrm{s},\left(\mathrm{CH}_{3}\right)_{2} \mathrm{~S}^{+}\right)$, 2.10(3H, s, tosyl $\left.\mathrm{CH}_{3}\right), 1.68 \sim 1.90\left(4 \mathrm{H}, \mathrm{m}, \mathrm{CH}_{2} \mathrm{CH}_{2} \mathrm{CH}_{2} \mathrm{CH}_{2}\right)$. Anal Calcd for $\mathrm{C}_{19} \mathrm{H}_{26} \mathrm{O}_{4} \mathrm{~S}_{2}: \mathrm{C}, 59.66$; H, 6.85. Found: C, 59.69; H, 7.00.

Dimethyl(5-phenoxypentyl)sulfonium $\boldsymbol{p}$-toluenesulfonate (12): $\mathrm{mp} \quad 143-144{ }^{\circ} \mathrm{C} ;{ }^{1} \mathrm{H}-\mathrm{NMR}$ $\left(\mathrm{DMSO}_{-} \mathrm{d}_{6}\right) \delta: \quad 7.34\left(2 \mathrm{H}, \mathrm{d}, \mathrm{J}=7.8 \mathrm{~Hz}\right.$, tosyl $\left.H_{3,5}\right), 6.95\left(2 \mathrm{H}, \mathrm{d}, \mathrm{J}=7.8 \mathrm{~Hz}\right.$, tosyl $\left.H_{2,6}\right), 6.65 \sim 7.25(5 \mathrm{H}$, $\mathrm{m}$, aromatic $H), 3.82\left(2 \mathrm{H}, \mathrm{t}, \mathrm{J}=6.0 \mathrm{~Hz}, \mathrm{CH}_{2} \mathrm{O}\right), 3.17\left(2 \mathrm{H}, \mathrm{t}, \mathrm{J}=6.0 \mathrm{~Hz}, \mathrm{~S}^{+} \mathrm{CH}_{2}\right), 2.78\left(6 \mathrm{H}, \mathrm{s},\left(\mathrm{CH}_{3}\right)_{2} \mathrm{~S}^{+}\right)$, 2.16(3H, s, tosyl $\left.\mathrm{CH}_{3}\right), 1.40 \sim 1.80\left(6 \mathrm{H}, \mathrm{m}, \mathrm{CH}_{2} \mathrm{CH}_{2} \mathrm{CH}_{2} \mathrm{CH}_{2} \mathrm{CH}_{2}\right)$. Anal Calcd for $\mathrm{C}_{20} \mathrm{H}_{28} \mathrm{O}_{4} \mathrm{~S}_{2}: \mathrm{C}$, 60.57; H, 7.12. Found: C, 60.34; H, 7.21.

1-Methyl-2-phenoxyethyldimethylsulfonium $\quad$-toluenesulfonate $\quad(13): \quad m p \quad 48-50 \quad{ }^{\circ} \mathrm{C}$; ${ }^{1} \mathrm{H}-\mathrm{NMR}\left(\mathrm{DMSO}_{\mathrm{d}}\right) \delta$ : 7.51(2H, d, J=7.8Hz, tosyl $\left.H_{3,5}\right), 7.11\left(2 \mathrm{H}, \mathrm{d}, \mathrm{J}=7.8 \mathrm{~Hz}\right.$, tosyl $\left.H_{2,6}\right)$, 6.80 7.35(5H, m, aromatic H), 4.30(2H, m, $\left.\mathrm{CH}_{2} \mathrm{O}\right), 3.95\left(1 \mathrm{H}, \mathrm{m}, \mathrm{S}^{+} \mathrm{CH}\right), 2.94\left(3 \mathrm{H}, \mathrm{s}, \mathrm{CH}_{3} \mathrm{~S}^{+}\right)$, $2.90\left(3 \mathrm{H}, \mathrm{s}, \mathrm{CH}_{3} \mathrm{~S}^{+}\right), 2.26\left(3 \mathrm{H}, \mathrm{s}\right.$, tosyl $\left.\mathrm{CH}_{3}\right), 1.48\left(3 \mathrm{H}, \mathrm{d}, \mathrm{J}=6.4 \mathrm{~Hz}, \mathrm{CHCH}_{3}\right)$. Anal Calcd for $\mathrm{C}_{18} \mathrm{H}_{24} \mathrm{O}_{4} \mathrm{~S}_{2}$ : C, 58.67; H, 6.56. Found: C, 58.38; H, 6.81.

2-Methyl-2-phenoxyethyldimethylsulfonium $\quad \boldsymbol{p}$-toluenesulfonate $\quad(14)$ : $\quad$ oil. $\quad{ }^{1} \mathrm{H}-\mathrm{NMR}$ $\left(\mathrm{DMSO}_{-} \mathrm{d}_{6}\right) \delta: 7.51\left(2 \mathrm{H}, \mathrm{d}, \mathrm{J}=7.8 \mathrm{~Hz}\right.$, tosyl $\left.H_{3,5}\right), 7.14\left(2 \mathrm{H}, \mathrm{d}, \mathrm{J}=7.8 \mathrm{~Hz}\right.$, tosyl $\left.H_{2,6}\right), 6.90 \sim 7.40(5 \mathrm{H}, \mathrm{m}$, aromatic $H), 4.99(1 \mathrm{H}, \mathrm{m}, \mathrm{CHO}), 3.72\left(2 \mathrm{H}, \mathrm{m}, \mathrm{S}^{+} \mathrm{CH}_{2}\right), 2.99\left(3 \mathrm{H}, \mathrm{s}, \mathrm{CH}_{3} \mathrm{~S}^{+}\right), 2.95\left(3 \mathrm{H}, \mathrm{s}, \mathrm{CH}_{3} \mathrm{~S}^{+}\right)$, $2.28\left(3 \mathrm{H}, \mathrm{s}\right.$, tosyl $\left.\mathrm{CH}_{3}\right), 1.34\left(3 \mathrm{H}, \mathrm{d}, \mathrm{J}=6.1 \mathrm{~Hz}, \mathrm{CHCH}_{3}\right)$. Anal Calcd for $\mathrm{C}_{18} \mathrm{H}_{24} \mathrm{O}_{4} \mathrm{~S}_{2}: \mathrm{C}, 58.67 ; \mathrm{H}$, 6.56. Found: C, 58.42; H, 6.85 .

1-Methyl-3-phenoxytetrahydrothiopyranium $\boldsymbol{p}$-toluenesulfonate (cis:trans=1:1) (15): $\mathrm{mp}$ 106-107.5 ${ }^{\circ} \mathrm{C}$; 1H-NMR (DMSO-d $\left.{ }_{6}\right) \delta: \quad 7.49\left(2 \mathrm{H}, \mathrm{d}, \mathrm{J}=7.8 \mathrm{~Hz}\right.$, tosyl $\left.H_{3,5}\right), 7.03(2 \mathrm{H}, \mathrm{d}, \mathrm{J}=7.8 \mathrm{~Hz}$, tosyl $\left.H_{2,6}\right), 6.90 \sim 7.40(5 \mathrm{H}, \mathrm{m}$, aromatic $H), 4.35(1 \mathrm{H}, \mathrm{m}, \mathrm{CHO}), 3.70 \sim 3.80\left(4 \mathrm{H}, \mathrm{m}, \mathrm{CH}_{2} \mathrm{~S}^{+} \mathrm{CH}_{2}\right)$, 2.91( $\left(3 \mathrm{H}, \mathrm{s}, \mathrm{CH}_{3} \mathrm{~S}^{+}\right), 3.00\left(3 \mathrm{H}, \mathrm{s}, \mathrm{CH}_{3} \mathrm{~S}^{+}\right), 2.24\left(3 \mathrm{H}, \mathrm{s}\right.$, tosyl $\left.\mathrm{CH}_{3}\right), 1.85\left(4 \mathrm{H}, \mathrm{m}, \mathrm{S}^{+} \mathrm{CH}_{2} \mathrm{CH}_{2} \mathrm{CH}_{2}\right)$. Anal Calcd for $\mathrm{C}_{19} \mathrm{H}_{24} \mathrm{O}_{4} \mathrm{~S}_{2}$ : C, 59.97; $\mathrm{H}, 6.36$. Found: $\mathrm{C}, 59.72 ; \mathrm{H}, 6.46$.

Dimethyl(3-phenylpropyl) sulfonium p-toluenesulfonate (16): $\mathrm{mp} 137.5-138.5{ }^{\circ} \mathrm{C} ;{ }^{1} \mathrm{H}-\mathrm{NMR}$ $\left(\mathrm{DMSO}_{-}\right) \delta$ : $7.38\left(2 \mathrm{H}, \mathrm{d}, \mathrm{J}=7.8 \mathrm{~Hz}\right.$, tosyl $\left.H_{3,5}\right), 7.00\left(2 \mathrm{H}, \mathrm{d}, \mathrm{J}=7.8 \mathrm{~Hz}\right.$, tosyl $\left.H_{2,6}\right), 6.75 \sim 7.30(5 \mathrm{H}, \mathrm{m}$, aromatic H), 3.60(2H, t, J=6.0Hz, $\left.\mathrm{S}^{+} \mathrm{CH}_{2} \mathrm{CH}_{2} \mathrm{CH}_{2}\right), 3.50\left(2 \mathrm{H}, \mathrm{t}, \mathrm{J}=6.0 \mathrm{~Hz}, \mathrm{~S}^{+} \mathrm{CH}_{2}\right), 2.89(6 \mathrm{H}, \mathrm{s}$, $\left.\left(\mathrm{CH}_{3}\right)_{2} \mathrm{~S}+\right), 2.25\left(3 \mathrm{H}\right.$, s, tosyl $\left.\mathrm{CH}_{3}\right), 1.76\left(2 \mathrm{H}, \mathrm{m}, \mathrm{S}^{+} \mathrm{CH}_{2} \mathrm{CH}_{2} \mathrm{CH}_{2}\right)$, Anal Calcd for $\mathrm{C}_{18} \mathrm{H}_{24} \mathrm{O}_{3} \mathrm{~S}_{2}: \mathrm{C}$, 
61.33; H, 6.86. Found: C, 61.14; H, 6.77.

Dimethyl(2-phenylthioethyl)sulfonium $p$-toluenesulfonate (17): $\mathrm{mp} \quad 118-119{ }^{\circ} \mathrm{C} ;{ }^{1} \mathrm{H}$ NMR (DMSO-d $\left.\mathrm{d}_{6}\right) \delta: 7.51\left(2 \mathrm{H}, \mathrm{d}, \mathrm{J}=7.8 \mathrm{~Hz}\right.$, tosyl $\left.H_{3,5}\right), 7.23 \sim 7.43(5 \mathrm{H}, \mathrm{m}$, aromatic $\mathrm{H}), 7.11(2 \mathrm{H}, \mathrm{d}$, $\mathrm{J}=7.8 \mathrm{~Hz}$, tosyl $\left.\mathrm{H}_{2,6}\right), 3.51\left(2 \mathrm{H}, \mathrm{t}, \mathrm{J}=5.3 \mathrm{~Hz}, \mathrm{~S}^{+} \mathrm{CH}_{2}\right), 3.49\left(2 \mathrm{H}, \mathrm{t}, \mathrm{J}=5.3 \mathrm{~Hz}, \mathrm{~S}^{+} \mathrm{CH}_{2} \mathrm{CH}_{2}\right), 2.93(6 \mathrm{H}, \mathrm{s}$, $\left.\left(\mathrm{CH}_{3}\right)_{2} \mathrm{~S}+\right), 2.29\left(3 \mathrm{H}\right.$, s, tosyl $\left.\mathrm{CH}_{3}\right)$. Anal Calcd for $\mathrm{C}_{17} \mathrm{H}_{22} \mathrm{O}_{3} \mathrm{~S}_{3}: \mathrm{C}, 55.10 ; \mathrm{H}, 5.98$. Found: C, 55.09; $\mathrm{H}, 5.94$.

\subsection{Homologous Passive Cutaneous Anaphylaxis (PCA) in Rat}

Antiserum containing homocytotropic antibody was obtained from rats that had been immunized with 2,4-dinitrophenyl-coupled ascaris (DNP-As) mixed with killed Bordetella pertussis according to the method of Tada et al.[4] The antibody titer of this serum (rat anti-DNP-As serum ) was about 1:256 as estimated by the $48 \mathrm{~h}$ PCA. The antiserum diluted 20 -fold with $0.9 \%$ saline was injected intradermally as $0.1 \mathrm{~mL}$ dose into 3 sites on the shaved backs of normal rats (The number of animals is 7 or 8). The same dose of physiologic saline was similarly injected into the other side. After $48 \mathrm{hr}, 1.0 \mathrm{~mL}$ of $0.25 \%$ Evans blue solution containing $2.0 \mathrm{mg}$ of antigen were given to the animals intravenously. Thirty minutes later, the animals were killed by exsanguination and the skins were removed to measure the PCA bluing lesion. The amount of the dye was then estimated colormetrically after extraction by the method of Harada [8].

The authors are grateful to Prof. Tadashi Kataoka and Dr. Hiroshi Shimizu (Gifu Pharmaceutical University) for useful discussions and valuable suggestions.

\section{References}

[1] A. J. Cunningham, and A. Szongerg, Immunology, 14, 599-600 (1968).

[2] Y. Tada, I. Yamawaki, S. Ueda, H. Matsumoto, N. Matsuura, M. Yasumoto, A. Koda, and M. Hori, Chem-Bio. Info. J. 1, 51-59, (2001).

[3] Y. Tada, I. Yamawaki, S. Ueda, H. Matsumoto, N. Matsuura, M. Yasumoto, A. Koda, M. Hori, J. Med. Chem., 41, 3330-3336 (1998).

[4] T. Tada, and K. Okamura, J. Immunol. 106, 1002-1011 (1971).

[5] A. Koda, H. Nagai, S. Watanabe, Y. Yanagihara, K. Sakamoto, J. Allergy Clin. Immunol., 57, 396-407 (1976).

[6] J. Crosby, and . J. M. Stirling, J. Amer. Chem. Soc., 90, 6869-6870 (1969).

[7] A. J. Leo, Chemical Reviews, 4, 1281-1305 (1993).

[8] K. Fukui, C. Nagata, and A. Imamura, Science, 132, 87-89 (1960).

[9] J. J. P. Stewart, J. Comput. Chem., 10, 209-220 (1989).

[10] M. Harada, T. Takeuchi, and T. Katagiri, Dermatologica, 142, 193-201 (1971). 